\title{
MOLECULAR DYNAMICS STUDY ON STRUCTURAL RELAXATION OF METALLIC GLASSES
}

\author{
MASATO Shimono ${ }^{a}$ and HIDEHIRO Onodera ${ }^{b}$ \\ National Institute for Materials Science, 1-2-1 Sengen, Tsukuba, 305-0047, Japan \\ aemail: shimono.masato@nims.go.jp \\ bemail: hidehiro.onodera@nims.go.jp
}

Keywords: Metallic Glasses, Glass transition, Structural Relaxation, Rapid Solidification.

\begin{abstract}
Structural relaxation process in the $\mathrm{Zr}-\mathrm{Cu}$ metallic glasses is investigated by using molecular dynamics simulations. The enthalpy change in isothermal annealing of the glassy state cannot be fitted by a simple exponential function but obeys a stretched exponential function, which indicates that the relaxation in glassy phase is not a single Debye type process. A close examination of individual atomic motion reveals that the enthalpy relaxation is related to a string-like cooperative motion of atoms. The analysis of the local symmetry around each atom shows that a network of the icosahedral clusters grows in the glassy phases during annealing and it closely relates to the free-volume annihilation in the structural relaxation.
\end{abstract}

\section{Introduction}

Glass transition always has a kinetic nature. Structure and all physical properties such as enthalpy, entropy and volume are frozen in due to extreme slowdown of atomic mobility at the glass transition point. Therefore, by isothermal annealing at finite temperature, any metallic glass $[1,2]$ obtained in laboratories undergoes a structural change towards some metastable equilibrium structure, which is called structural relaxation. Generally, the structural relaxation is accompanied by free-volume annihilation in the glassy state. However, the atomistic mechanism that causes the free-volume annihilation is not understood at all, because such microscopic behavior is difficult to investigate by experimental methods. On the other hands, molecular dynamics (MD) simulation is a powerful tool to understand the atomistic mechanism of dynamical processes such as the structural relaxation. So in the present study, structural relaxation processes in glassy phases of the $\mathrm{Zr}-\mathrm{Cu}$ binary system are investigated by using MD simulations. In the simulation, we pay special attention on the enthalpy change and the free-volume reduction in isothermal annealing, the atomic motions during the relaxation process, and the change of local icosahedral symmetry in the annealed glass.

\section{Method}

As an interatomic potential we use a many-body Finnis-Sinclare type potential [3] cultivated by Rosato et al. [4], which has a functional form as

$$
\begin{aligned}
& E_{i}=-\sqrt{\rho_{i}}+\sum_{j} \phi_{i j}, \\
& \phi_{i j i}\left(r_{i j}\right)=A_{i j} \exp p_{i j}\left(1-r_{i j} / r_{i j}{ }^{0}\right), \\
& \rho_{i}\left(r_{i j}\right)=\sum_{j} \xi_{i j}^{2} \exp 2 q_{i j}\left(1-r_{i j} / r_{i j}{ }^{0}\right),
\end{aligned}
$$


where $r_{i j}$ is the distance between atoms $i$ and $j$ and the parameters $p_{i j}, q_{i j}, A_{i j}, \xi_{i j}$ and $r_{i j}{ }^{0}$ are determined by us to reproduce the mixing enthalpy of the $\mathrm{Zr}-\mathrm{Cu}$ system and the lattice parameters, elastic moduli and the cohesive energies of hcp- $\mathrm{Zr}$, fcc- $\mathrm{Cu}$, and the $\mathrm{B} 2-\mathrm{ZrCu}$ phase as $p_{\mathrm{CuCu}}=10.712, p_{\mathrm{CuZr}}=10.241$, $p_{\mathrm{ZrZr}}=9.771, q_{\mathrm{CuCu}}=2.424, q_{\mathrm{CuZr}}=2.232, q_{\mathrm{ZrZr}}=2.039, A_{\mathrm{CuCu}}=0.08851 \mathrm{eV}, A_{\mathrm{CuZr}}=0.1280 \mathrm{eV}, A_{\mathrm{ZrZr}}$ $=0.1397 \mathrm{eV}, \xi_{\mathrm{CuCu}}=1.2889 \mathrm{eV}, \xi_{\mathrm{CuZr}}=1.7700 \mathrm{eV}, \xi_{\mathrm{ZZZr}}=2.1706 \mathrm{eV}, r_{\mathrm{CuCu}}{ }^{0}=0.2556 \mathrm{~nm}, r_{\mathrm{CuZr}}{ }^{0}=$ $0.2830 \mathrm{~nm}$ and $r_{\mathrm{ZrZr}}{ }^{0}=0.3205 \mathrm{~nm}$.

The simulation system consists of 4000-16000 atoms. The system is kept at zero pressure during the simulation by using the Parrinello-Rahman scheme [5] or the Andersen scheme [6]. The system temperature is controlled by rescaling the atomic momenta. The equations of motion are numerically solved by the 5th-order Gear's method [7] and the integration time step is $1.2 \mathrm{fs}$.

\section{Results}

In the simulations, we first prepare an equilibrium liquid phase by holding the $\mathrm{Zr}$-Cu mixture at a sufficiently high temperature, and then obtain a glassy phase by quenching the system rapidly. Main calculations are performed on the $\mathrm{Zr}-\mathrm{Cu}$ binary system with the composition range from $40 \%$ to $60 \%$ at. $\mathrm{Cu}$.

The temperature dependence of the atomic enthalpy in two cooling procedures from a liquid phase of the $\mathrm{Zr}_{50} \mathrm{Cu}_{50}$ system is shown in Fig. 1. We can identify the glass transition temperature $T_{\mathrm{g}}$ by the jump of the slope of the temperature dependence of the enthalpy or the specific heat, as indicated in Fig. 1 by arrows. We can see that $T_{\mathrm{g}}$ depends on the cooling rate, because it is kinetically determined by the competition between the cooling rate and the relaxation time of the supercooled liquid phase. For the $\mathrm{Zr}_{50} \mathrm{Cu}_{50}$ system shown in Fig. 1, the estimated $T_{\mathrm{g}}$ is $750 \mathrm{~K}$ for the rapid cooling rate $(5 \mathrm{~K} / \mathrm{ps})$ and $710 \mathrm{~K}$ for the slow cooling rate $(0.5$ $\mathrm{K} / \mathrm{ps})$. Taking the extremely high cooling rates in the simulation into account, these values are consistent with the experimental observations [8] in this system.

After getting the glassy phases, we heat them up again, and keep them at a temperature just below $T_{\mathrm{g}}$ for isothermal annealing. The time evolution of the atomic enthalpy of the glassy phase in the annealing process is shown in Fig. 2 for the $\mathrm{Zr}_{50} \mathrm{Cu}_{50}$ system. The time dependence shows an apparent deviation from a simple Debye type behavior. So we try to fit the results by a stretched exponential function: $\Delta E(t)$ $=\Delta E_{0}\left[1-\exp \left\{-(t / \tau)^{\beta}\right\}\right]$. As shown in Fig. 2, the simulation data can be fitted well and the fitted parameters are $\beta=0.51$ and $\tau=3.9 \mathrm{~ns}$ for $T=580 \mathrm{~K}$ and $\beta=0.85$ and $\tau=0.48 \mathrm{~ns}$ for $T=700 \mathrm{~K}$. In both cases, the glassy state is far from its fully relaxed state at the corresponding temperature in the simulation time and the relaxation process is still going on, so the fitted values of $\beta$ and $\tau$ may change in a longer observation. However, it is quite interesting that this behavior has the same nature as has been found in the relaxation behavior of metallic glasses by

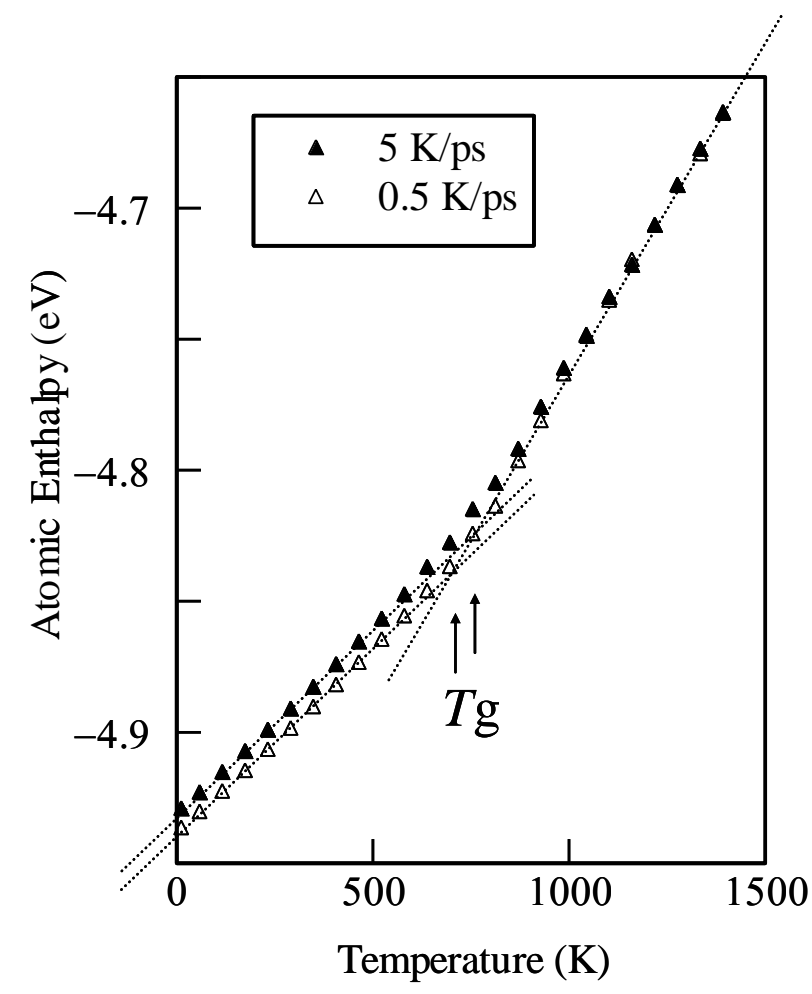

Fig. 1 The temperature dependence of the atomic enthalpy of the $\mathrm{Zr}_{50} \mathrm{Cu}_{50}$ system quenched from liquid state at cooling rate $5 \mathrm{~K} / \mathrm{ps}$ (closed triangles) and $0.5 \mathrm{~K} / \mathrm{ps}$ (open triangles). 
the experimental studies $[9,10,11$, 12], although the handled time-scales of observation are completely different between the experiments and the simulations.

The fact that the exponent $\beta$ is not unity indicates that there are two or more different relaxation processes with different relaxation times. So we have investigated the time evolution of the atomic enthalpy of the annealed glassy phase more closely. In Fig. 3 all enthalpy data averaged over every $1000 \mathrm{MD}$ steps for a $\mathrm{Zr}_{40} \mathrm{Cu}_{60}$ glassy alloy annealed at $700 \mathrm{~K}$ are shown by triangles for the beginning $1 \mathrm{~ns}$ annealing time in the linear scale. We can recognize two types of the typical behavior of the enthalpy change: one is a gradual and continuous decrease and the other is a sudden stepwise change. It suggests us that there should be two different types of relaxation procedure, that is, a gradual one and a sudden one.

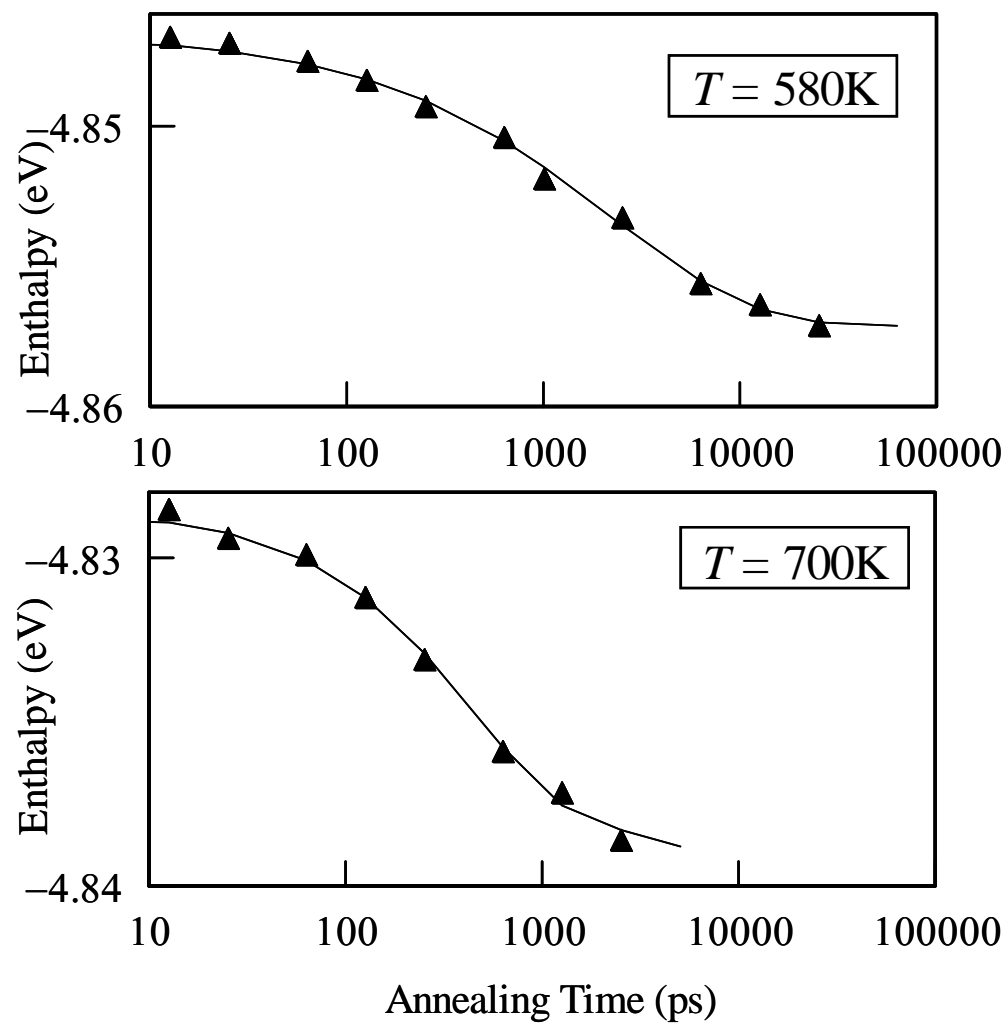

Fig. 2 The annealing time dependence of the atomic enthalpy of the $\mathrm{Zr}_{50} \mathrm{Cu}_{50}$ glassy phase annealed at $580 \mathrm{~K}$ (upper) and at $700 \mathrm{~K}$ (lower). Solid lines are the fitted curves by a stretched exponential function.

To clarify the atomistic mechanism in the relaxation procedure, we analyze the individual atomic motion during the "stepwise" behavior in the enthalpy change. We focus on a short time interval between the annealing time $t=784$ ps and 790 ps indicated by arrows in Fig.3, and then we search some characteristic feature in the diffusive motions of atoms in the interval. In Fig. 4 the atoms that have diffused at amount more than $60 \%$ of atomic size in the short period ( $6 \mathrm{ps})$ are depicted by the

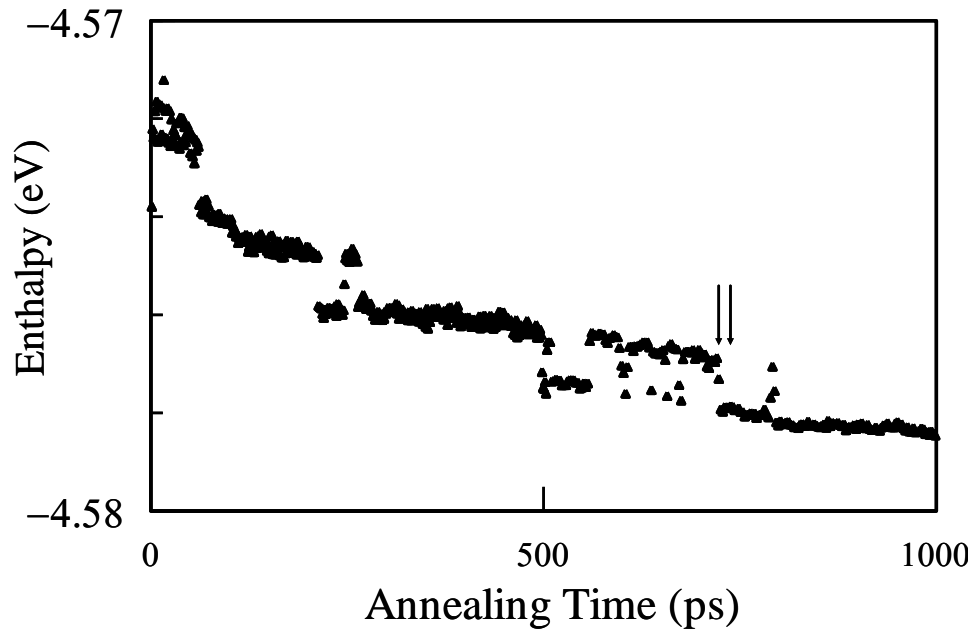

Fig. 3 The time evolution of the atomic enthalpy of the $\mathrm{Zr}_{40} \mathrm{Cu}_{60}$ glassy phase annealed at $700 \mathrm{~K}$ in the first $1 \mathrm{~ns}$ expressed in the linear scale.

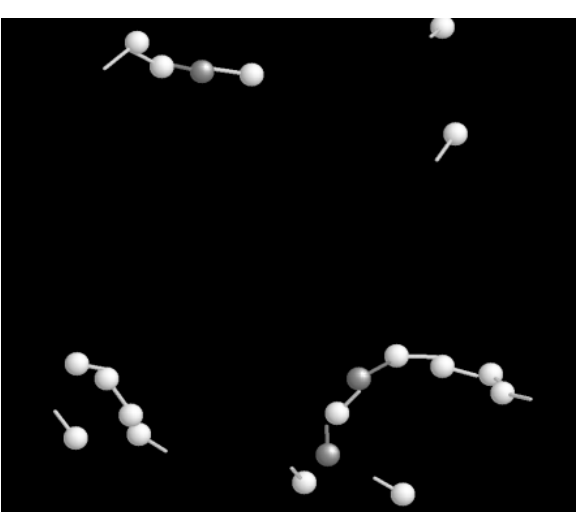

Fig. 4 A sectional snapshot of the atoms showing a considerable diffusion in the $\mathrm{Zr}_{40} \mathrm{Cu}_{60}$ glassy phase annealed at $700 \mathrm{~K}$. The dark gray and the light gray spheres denote $\mathrm{Zr}$ and $\mathrm{Cu}$ atoms, respectively, and the sticks denote their trajectories. 


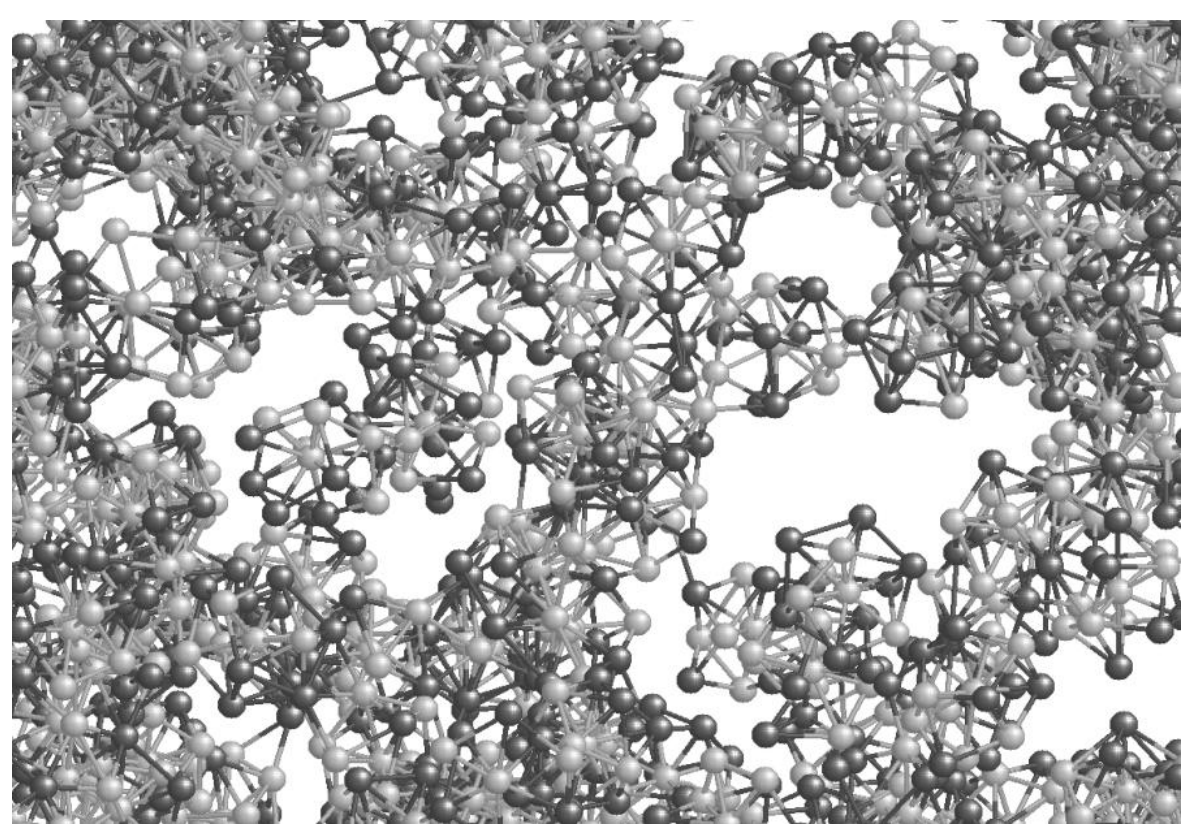

Fig. 5 A snapshot of the network structure of icosahedral clusters found in an as-quenched $\mathrm{Zr}_{40} \mathrm{Cu}_{60}$ glassy phase. The dark gray and the light gray spheres denote $\mathrm{Zr}$ and $\mathrm{Cu}$ atoms, respectively, and only the atoms forming the icosahedral clusters are depicted.

spheres and their trajectories are depicted by the sticks. Among hopping-like motions of a single atom, we can recognize a string-like cooperative motion of several atoms. Therefore, the string-like cooperative motion might contribute to the "sudden" process of relaxation and should play an important role in the relaxation mechanism of glassy phases.

Both experimental observations [13] and simulation studies [14, 15] suggest that the icosahedral symmetry should be a key factor to understand the atomic structure of metallic glasses. So we analyze the icosahedral symmetry in the $\mathrm{Zr}-\mathrm{Cu}$ glassy phases obtained in the simulations by using the Voronoi tessellation technique [14]. As depicted in Fig. 5, we have observed a network structure $[16,17]$ of icosahedral clusters in an as-quenched $\mathrm{Zr}_{40} \mathrm{Cu}_{60}$ glassy phase. Moreover, as shown in Fig. 6 , the density of the icosahedral clusters increases in the course of isothermal annealing at $T=700 \mathrm{~K}$, and hence their network also grows during annealing. Therefore, free-volume annihilation in the structural relaxation in this system should be always accompanied with growth of the icosahedral network, because the icosahedral cluster is one of the most effective and dense packing structure of atoms.

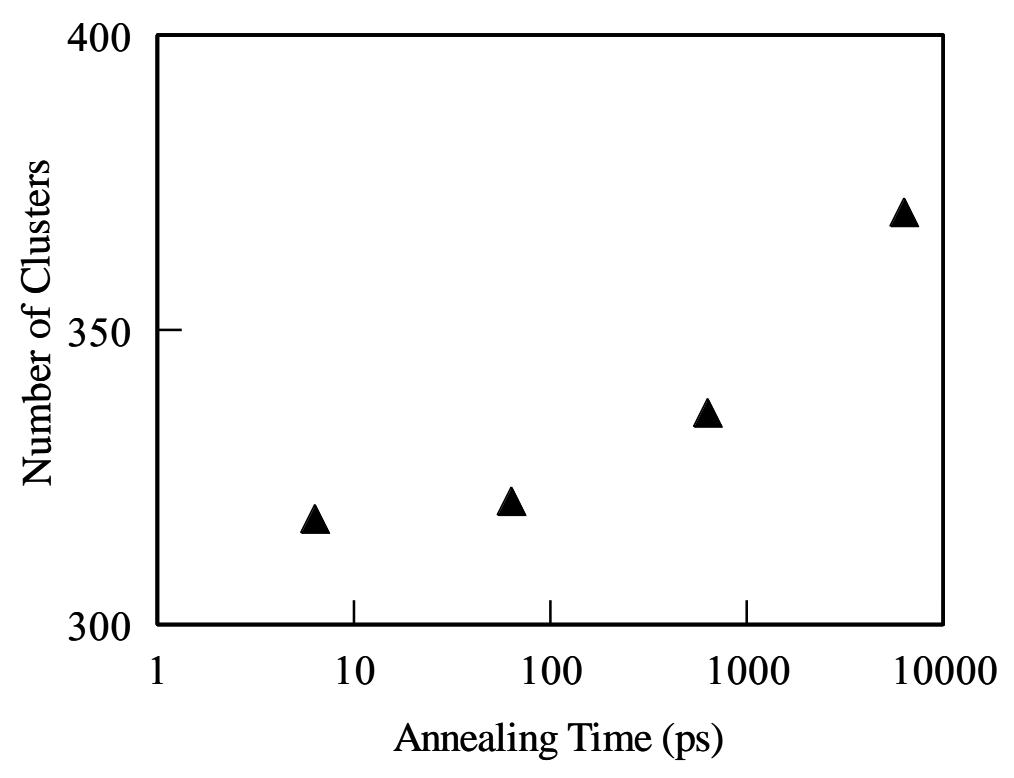

Fig. 6 The annealing time dependence of the number of the icosahedral clusters in the $\mathrm{Zr}_{40} \mathrm{Cu}_{60}$ glassy phase annealed at $700 \mathrm{~K}$ 


\section{Summary}

The structural relaxation procedure of the $\mathrm{Zr}-\mathrm{Cu}$ metallic glasses is investigated by using molecular dynamics simulations. The time evolution of the enthalpy of annealed glassy state shows a non-Debye behavior that can be fitted to a stretched exponential form. The analysis of the atomic motions during the annealing process suggests that a string-like cooperative motion should be closely related to the relaxation process. A network structure of the icosahedral clusters is formed in glassy states and it grows as the relaxation goes on, and its growth causes the free-volume reduction in the glassy phases.

\section{References}

[1] A. Inoue, T. Zang and T. Masumoto: Mater. Trans. JIM 31 (1990), p. 177.

[2] A. Parker and W. L. Johnson: Appl. Phys. Lett. 63 (1993), p. 2342.

[3] M. W. Finnis and J. E. Sinclair: Pil. Mag. 50 (1984), p. 45.

[4] V. Rosato, M. Guillope and B. Legrand: Phil. Mag. 59 (1989), p. 321.

[5] M. Parrinello and A. Rahman: Phys. Rev. Lett. 45 (1980), p. 1196.

[6] H.C. Andersen: J. Chem. Phys. 72 (1980), p. 2384.

[7] C.W. Gear: Numerical Initial Value Problems in Ordinary Differential Equations (Prentice-Hall, New Jersey 1971)

[8] T. Ichitsubo, E. Matasubara and H. Numakura: Mater. Sci. Eng. A 449 (2007), p. 506.

[9] R. Busch, E. Bakke and W.L. Johnson: Acta Mater. 46 (1998), p. 4725.

[10] G.J. Fan, J.F. Loffler, R.K. Wunderlich and H.-J. Fecht: Acta Mater. 52 (2004), p. 667

[11] O. Haruyama, Y. Yokoyama and A. Inoue: Mater. Trans. 48 (2007), p. 1708.

[12] A. Ishii, F. Hori, A. Iwase, Y. Fukumoto, Y. Yokoyama and T.J. Konno: Mater. Trans. 49 (2008), p. 1975.

[13] J. Saida, T. Sanada, S. Sato, M. Imafuku, E. Matsubara and A. Inoue: Mater. Trans. 48 (2007), p. 1703.

[14]F. Yonezawa, Solid State Physics Vol. 45, Academic Press, San Diego, (1991), p.179.

[15] M. Shimono and H. Onodera: Mater. Trans. JIM 39 (1998), p. 147.

[16]H.W. Sheng, W.K. Luo, F.M. Alamgir, J.M. Bai and E. Ma: Nature 439 (2006), p. 419.

[17] M. Shimono and H. Onodera: Mater. Sci. Forum Vol. 539-543 (2007), p. 2031. 